\title{
A STABILITY CRITERION FOR BIORTHOGONAL WAVELET BASES AND THEIR RELATED SUBBAND CODING SCHEME
}

\section{ALBERT COHEN AND INGRID DAUBECHIES}

Introduction. In a recent work with J. C. Feauveau [CDF], we introduced biorthogonal bases of compactly supported wavelets, i.e., pairs of dual Riesz bases generated from two single compactly supported functions $\psi$ and $\tilde{\psi}$ by means of dilations and translations

$$
\begin{cases}\psi_{k}^{j}(x)=2^{-j / 2} \psi\left(2^{-j} x-k\right) & (j, k) \in \mathbb{Z}^{2} \\ \tilde{\psi}_{k}^{j}(x)=2^{-j / 2} \tilde{\psi}\left(2^{-j} x-k\right) & (j, k) \in \mathbb{Z}^{2} .\end{cases}
$$

This construction mimics, in a more general setting, the construction of orthonormal bases of compactly supported wavelets developed in [Dau1] that we briefly recall here in three steps:

- Orthonormal wavelets are associated with a scaling function $\varphi$ which defines a multiresolution analysis, i.e., a ladder of embedded approximation subspaces of $L^{2}(\mathbb{R})$

$$
\{0\} \rightarrow \cdots V_{1} \subset V_{0} \subset V_{-1} \cdots \rightarrow L^{2}(\mathbb{R})
$$

such that $\left\{\varphi_{k}^{j}\right\}_{k \in \mathbb{Z}}=\left\{2^{-j / 2} \varphi\left(2^{-j} x-k\right)\right\}_{k \in \mathbb{Z}}$ is an orthonormal basis for $V_{j}$. The wavelets are built to characterize the missing details between two adjacent levels of approximation. More precisely, $\left\{\psi_{k}^{j}\right\}_{k \in \mathbb{Z}}=\left\{2^{-j / 2} \psi\left(2^{-j} x-k\right)\right\}_{k \in \mathbb{Z}}$ is an orthonormal basis for the orthogonal complement $W_{j}$ of $V_{j}$ in $V_{j-1}$.

- The constructions of $\varphi$ and $\psi$ are based on a trigonometric polynomial $m_{0}(\omega)$ such that $m_{0}(0)=1$ and

$$
\left|m_{0}(\omega)\right|^{2}+\left|m_{0}(\omega+\pi)\right|^{2}=1 .
$$

The functions $\varphi$ and $\psi$ are then defined by

$$
\hat{\varphi}(\omega)=\prod_{k=1}^{+\infty} m_{0}\left(2^{-k} \omega\right)
$$

and

$$
\hat{\psi}(\omega)=m_{1}\left(\frac{\omega}{2}\right) \hat{\varphi}\left(\frac{\omega}{2}\right)=e^{-i \omega / 2} \overline{m_{0}\left(\frac{\omega}{2}+\pi\right)} \hat{\varphi}\left(\frac{\omega}{2}\right) .
$$

Received 18 May 1992. 
It has been shown in [Co1] and [La] that, for a generic choice of $m_{0}(\omega)$, these formulas lead indeed to a scaling function which satisfies $\langle\varphi(x-k) \mid \varphi(x-l)\rangle=\delta_{k, l}$ and to an orthonormal wavelet basis.

- In the fast wavelet transform algorithm, $m_{0}$ and $m_{1}$ are the transfer functions of a low-pass and a high-pass filter that split the discrete signal into two channels. These channels are decimated (only one sample out of two is retained), and the process is iterated on the low-pass channel. The final result is a coarse approximation of the signal and a succession of details at each intermediate scale. Perfect reconstruction is performed by the same filters which are used to interpolate and refine the decimated channels.

Unfortunately, these filters, known as conjugate quadrature filters (CQF, see [SB]), have some disadvantages for practical design and applications:

- They cannot be both FIR and linear phase (i.e., with real and symmetrical coefficient) except for the uninteresting Haar filter (which leads to a discontinuous wavelet).

- They are solutions of the quadratic equation (1.8), and their coefficients are usually algebraic numbers with no simple expression.

- Their design uses the Féjer-Riesz factorization lemma which does not generalize in a multidimensional situation.

- When these filters have finite impulse response, the subspaces $V_{j}$ have no simple direct definition. For example, they cannot be spline function spaces, except in the Haar case.

For all these reasons, we considered a larger class of filters called "dual filters", as introduced in the work of Vetterli [Ve]; see also [VH]. The generalization is made by allowing the reconstruction and analysis filter to be different from each other. All the disadvantages of the CQF, as listed above, disappear, but we have lost orthonormality. These filters are still associated with a wavelet basis $\psi_{k}^{j}$, but this is not an orthonormal basis, and one needs a dual basis of the same type $\tilde{\psi}_{k}^{j}$ to compute the coordinates of any $L^{2}$ function by an inner product:

$$
f=\sum_{j, k \in \mathbb{Z}}\left\langle f \mid \tilde{\psi}_{k}^{j}\right\rangle \psi_{k}^{j} .
$$

An important problem is then to check that $\psi_{k}^{j}$ and $\tilde{\psi}_{k}^{j}$ are unconditional (or Riesz) bases, i.e., that the $L^{2}$ norm of a function is equivalent to the $l^{2}$ norm of the corresponding sequence of coefficients.

This problem can be formulated in subband coding terminology: the pyramidal algorithm decomposes a discrete signal $S_{0}$ into a coarse approximation $S_{J}$ and a sequence of additional details $\left\{D_{j}\right\}_{1 \leqslant j \leqslant J}$ in a recursive fashion, as described in Figure 1.

Can we say that this algorithm and its converse, the reconstruction process, are stable in the $l^{2}$ sense? We need to find two strictly positive constants $C_{1}$ and $C_{2}$ such that, independently of the choice of $J$,

$$
C_{1}\left\|S_{0}\right\|^{2} \leqslant\left\|S_{J}\right\|_{l^{2}}^{2}+\sum_{j=1}^{J}\left\|D_{j}\right\|_{l^{2}}^{2} \leqslant C_{2}\left\|S_{0}\right\|^{2}
$$




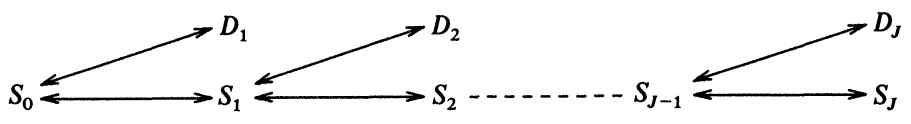

Figure 1. Multiscale decomposition of a discrete signal $S$

Since we have

$$
\sum_{k \in \mathbb{Z}} S_{0}(k) \varphi(x-k)=\sum_{k \in \mathbb{Z}} S_{J}(k) \varphi_{k}^{J}(x)+\sum_{j=1}^{J} \sum_{k \in \mathbb{Z}} D_{j}(k) \psi_{k}^{j}(x),
$$

the stability of the decomposition reconstruction algorithm will be achieved if and only if $\{\varphi(x-k)\}_{k \in \mathbb{Z}}$ and $\left\{\psi_{k}^{j}\right\}_{j, k \in \mathbb{Z}}$ are Riesz bases. By duality, the functions $\tilde{\varphi}$ and $\tilde{\psi}$ will satisfy the same property.

We shall see that, contrary to the orthonormal case, a pair of dual filters does not "almost always" lead to a pair of stable biorthogonal wavelet bases. We shall need special assumptions based on the transition operators associated to these filters.

These operators will be defined, and their main properties will be studied in Section 3. The main results, in particular the necessary and sufficient conditions for stability, are presented in Sections 4 and 5. (In [CDF] we gave a sufficient condition using stronger assumptions.)

We start by recalling some properties of dual filters and biorthogonal wavelets. For detailed proofs of the results presented in the next section, we refer to [CDF], [Co2], or [Dau2].

2. The construction of biorthogonal wavelets. The starting point in the construction of biorthogonal wavelets is a two-channel subband coding scheme with perfect reconstruction. It can be represented as in Figure 2. The original discrete signal is divided into two channels by the action of two discrete filters represented by their transfer functions $\overline{\tilde{m}_{0}(\omega)}$ (low-pass) and $\overline{\tilde{m}_{1}(\omega)}$ (high-pass), followed by a decimation of one sample out of two.

The reconstruction is made by interleaving zeros in these two subsampled channels and convolving with the filters $m_{0}(\omega)$ (low-pass) and $m_{1}(\omega)$ (high-pass). The equations of perfect reconstruction coupled with the constraint of finite-impulse response lead to the relations

$$
\begin{aligned}
& m_{1}(\omega)=e^{-i \omega} \overline{\tilde{m}_{0}(\omega+\pi)}, \\
& \tilde{m}_{1}(\omega)=e^{-i \omega} \overline{m_{0}(\omega+\pi)},
\end{aligned}
$$

and

$$
\overline{m_{0}(\omega)} \tilde{m}_{0}(\omega)+\overline{m_{0}(\omega+\pi)} \tilde{m}_{0}(\omega+\pi)=1
$$




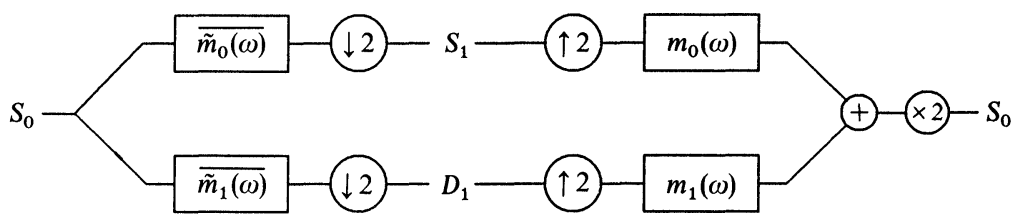

FIGURE 2. Perfect reconstruction subband coding scheme

(12) stands for downsampling, (12) for upsampling.

Note that when $m_{0}=\tilde{m}_{0}$ we find the CQF-orthonormal case presented in the introduction. We also assume that these filters satisfy

$$
m_{0}(0)=\tilde{m}_{0}(0)=1
$$

and

$$
m_{0}(\pi)=\tilde{m}_{0}(\pi)=0
$$

We then define the functions $\varphi, \psi, \tilde{\varphi}$, and $\tilde{\psi}$ by the formulas

$$
\begin{aligned}
& \hat{\varphi}(\omega)=\prod_{k=1}^{+\infty} m_{0}\left(2^{-k} \omega\right) \quad \text { and } \quad \hat{\psi}(\omega)=m_{1}\left(\frac{\omega}{2}\right) \hat{\varphi}\left(\frac{\omega}{2}\right) \\
& \hat{\varphi}(\omega)=\prod_{k=1}^{+\infty} \tilde{m}_{0}\left(2^{-k} \omega\right) \quad \text { and } \quad \hat{\psi}(\omega)=\tilde{m}_{1}\left(\frac{\omega}{2}\right) \hat{\varphi}\left(\frac{\omega}{2}\right)
\end{aligned}
$$

The infinite products converge because of (2.4); the resulting $\hat{\varphi}, \hat{\hat{\varphi}}$ are polynomially bounded, so that they are tempered distributions. Because $m_{0}(\omega), \tilde{m}_{0}(\omega)$ are trigonometric polynomials, $\varphi, \tilde{\varphi}, \psi$, and $\tilde{\psi}$ are compactly supported, by the Paley-Wiener theorem.

A question that naturally arises is when these functions belong to $L^{2}(\mathbb{R})$. In the orthonormal case this is automatic as a direct consequence of the CQF identity (1.3). In this more general setting, square integrability may fail; we shall discuss this in the next section.

We start by two preliminary results with sketchy proofs. (Full details are given in $[\mathrm{CDF}],[\mathrm{Co} 2]$, or [Dau2].)

A first result is the decomposition of any function $f$ in $L^{2}(\mathbb{R})$ in terms of $\psi_{k}^{j}$.

Proposition 2.1. If $\varphi, \tilde{\varphi}, \psi$, and $\tilde{\psi}$ are in $L^{2}(\mathbb{R})$, then for all $J>0$ and all $f$ in $L^{2}(\mathbb{R})$,

$$
\sum_{k \in \mathbb{Z}}\left\langle f \mid \tilde{\varphi}_{k}^{-J}\right\rangle \varphi_{k}^{-J}=\sum_{k \in \mathbb{Z}}\left\langle f \mid \tilde{\varphi}_{k}^{J}\right\rangle \varphi_{k}^{J}+\sum_{j=1-J}^{J} \sum_{k \in \mathbb{Z}}\left\langle f \mid \tilde{\psi}_{k}^{j}\right\rangle \psi_{k}^{j},
$$


and letting $J$ tend to $+\infty$,

$$
f=\lim _{J \rightarrow+\infty} \sum_{j=-J}^{J} \sum_{k \in \mathbb{Z}}\left\langle f \mid \tilde{\psi}_{k}^{j}\right\rangle \psi_{k}^{j}
$$

(in the $L^{2}$ sense). The same properties hold if we exchange $(\varphi, \psi)$ and $(\tilde{\varphi}, \tilde{\psi})$.

Proof. The detailed proof can be found in [Co2] (Theorem 2.1). Since $\varphi$ is square integrable and compactly supported, the function

$$
m(\omega)=\sum_{l \in \mathbb{Z}}|\hat{\varphi}(\omega+2 l \pi)|^{2}
$$

is a trigonometric polynomial (its Fourier coefficients are the $c_{k}=\langle\varphi(x) \mid \varphi(x-k)\rangle$ ) and thus uniformly bounded. The same holds for $\tilde{\varphi}, \psi$, and $\tilde{\psi}$. This gives sense to the summations in $k$ in the above formulas (2.8) and (2.9); using the Poisson summation formula and Parseval's formula, one finds that, for all $j \in \mathbb{Z}$,

$$
\left\|\sum_{k \in \mathbb{Z}}\left\langle f \mid \tilde{\varphi}_{k}^{j}\right\rangle \varphi_{k}^{j}\right\|_{L^{2}} \leqslant\left[\left(\sum_{l \in \mathbb{Z}}|\hat{\varphi}(\omega+2 l \pi)|^{2}\right)\left(\sum_{l \in \mathbb{Z}}|\hat{\varphi}(\omega+2 l \pi)|^{2}\right)\right]^{1 / 2}\|f\|_{L^{2}} .
$$

Once this summation makes sense, it suffices to combine the perfect reconstruction condition (2.1), (2.2), and (2.3) with the definitions (2.6) and (2.7) of $\varphi, \tilde{\varphi}, \psi$, and $\tilde{\psi}$ to obtain, for all $j \in \mathbb{Z}$ and for all $f$ in $L^{2}(\mathbb{R})$,

$$
\sum_{k \in \mathbb{Z}}\left\langle f \mid \tilde{\varphi}_{k}^{j-1}\right\rangle \varphi_{k}^{j-1}=\sum_{k \in \mathbb{Z}}\left\langle f \mid \tilde{\varphi}_{k}^{j}\right\rangle \varphi_{k}^{j}+\sum_{k \in \mathbb{Z}}\left\langle f \mid \tilde{\psi}_{k}^{j}\right\rangle \psi_{k}^{j}
$$

which immediately leads to (2.8) by a "telescoping" argument.

When $J$ tends to $+\infty$, one finds

$$
\lim _{J \rightarrow+\infty}\left\|\sum_{k \in \mathbb{Z}}\left\langle f \mid \tilde{\varphi}_{k}^{J}\right\rangle \varphi_{k}^{J}\right\|_{L^{2}}=0
$$

as a consequence of $\varphi, \tilde{\varphi} \in L^{2}(\mathbb{R})$. On the other hand, the properties $\int \tilde{\varphi}=\hat{\varphi}(0)=1$ (because $\tilde{m}_{0}(0)=1$ ) and $\sum_{k \in \mathbb{Z}} \varphi(x-k)=1$ (because $m_{0}(\pi)=0 \Rightarrow \hat{\varphi}(2 k \pi)=\delta_{0, k}$ ) imply that

$$
\lim _{J \rightarrow-\infty}\left\|\sum_{k \in \mathbb{Z}}\left\langle f \mid \tilde{\varphi}_{k}^{J}\right\rangle \varphi_{k}^{J}-f\right\|_{L^{2}}=0
$$

The decomposition (2.9) follows.

A second result (Lemma 2.6 in [Co2] and Lemma 3.7 in [CDF]) tells us that the linear independence and the biorthogonality for the $\psi_{k}^{j}$ and $\tilde{\psi}_{k}^{j}$ are related to the type of convergence of the infinite products in (2.6) and (2.7). 
Proposition 2.2. If the approximants $\varphi_{n}(x)$ and $\tilde{\varphi}_{n}(x)$ defined by

$$
\begin{aligned}
& \hat{\varphi}_{n}(\omega)=\prod_{k=1}^{n} m_{0}\left(2^{-k} \omega\right) \chi_{\left[-2^{n} \pi, 2^{n} \pi\right]}(\omega), \\
& \hat{\tilde{\varphi}}_{n}(\omega)=\prod_{k=1}^{n} \tilde{m}_{0}\left(2^{-k} \omega\right) \chi_{\left[-2^{n_{\pi}, 2^{n}}\right]}(\omega),
\end{aligned}
$$

converge to $\varphi$ and $\tilde{\varphi}$ in $L^{2}(\mathbb{R})$ as $n$ tends to $+\infty$, then we have

$$
\langle\varphi(x-k) \mid \tilde{\varphi}(x-l)\rangle=\delta_{k, l},
$$

and this is equivalent to

$$
\left\langle\psi_{k}^{j} \mid \tilde{\psi}_{k^{\prime}}^{j^{\prime}}\right\rangle=\delta_{j, j^{\prime}} \delta_{k, k^{\prime}}
$$

Proof. By recursion on $n$ and using the identity (2.3), one shows that

$$
\left\langle\varphi_{n}(x-k) \mid \tilde{\varphi}_{n}(x-l)\right\rangle=\delta_{k, l},
$$

which leads to (2.17).

Using properties (2.1), (2.2), and (2.3) of the filters, one derives from (2.17) that

$$
\langle\psi(x-k) \mid \tilde{\psi}(x-l)\rangle=\delta_{k, l}
$$

and

$$
\langle\varphi(x-k) \mid \tilde{\psi}(x-l)\rangle=\langle\tilde{\varphi}(x-k) \mid \psi(x-l)\rangle=0 .
$$

These in turn imply (2.18). The equivalence of (2.17) and (2.18) can be shown by proving $(2.20) \Rightarrow(2.17)$.

Note that the duality relations (2.18) coupled with the decomposition (2.9) imply the linear independence of the families $\left\{\psi_{k}^{j}\right\}_{j, k \in \mathbb{Z}}$ and $\left\{\tilde{\psi}_{k}^{j}\right\}_{j, k \in \mathbb{Z}}$. The expansion in (2.9) is thus unique, but we do not know yet if it is stable, i.e., if the families $\left\{\psi_{k}^{j}\right\}_{j, k \in \mathbb{Z}}$ and $\left\{\tilde{\psi}_{k}^{j}\right\}_{j, k \in \mathbb{Z}}$ are Riesz bases.

Equation (2.17) implies that $\{\varphi(x-k)\}_{k \in \mathbb{Z}}$ and $\{\tilde{\varphi}(x-k)\}_{k \in \mathbb{Z}}$ are Riesz bases in the subspace that they generate, by the following argument. By definition, the set $\{\varphi(x-k)\}_{k \in \mathbb{Z}}$ is a Riesz basis for its closed linear span if there exist two constants $C_{2}>C_{1}>0$ such that, for all finite linear combinations of the $\varphi(x-k)$, we have

$$
C_{1} \sum\left|\alpha_{k}\right|^{2} \leqslant\left\|\sum \alpha_{k} \varphi(x-k)\right\|_{L^{2}}^{2} \leqslant C_{2} \sum\left|\alpha_{k}\right|^{2} .
$$

This is equivalent to

$$
C_{1} \leqslant \sum_{l \in \mathbb{Z}}|\hat{\varphi}(\omega+2 l \pi)|^{2} \leqslant C_{2}
$$


Since the $2 \pi$ periodic function $\sum_{l \in \mathbb{Z}}|\varphi(\omega+2 l \pi)|^{2}$ is in fact a trigonometric polynomial with coefficients $c_{k}=\langle\varphi(x) \mid \varphi(x-k)\rangle$, the upper bound in (2.23) always holds for $\varphi$ and $\tilde{\varphi}$. On the other hand, (2.19) can be rewritten as

$$
\sum_{l \in \mathbb{Z}} \overline{\hat{\varphi}(\omega+2 l \pi)} \hat{\varphi}(\omega+2 l \pi)=1,
$$

and thus by the Cauchy-Schwarz inequality

$$
\left(\sum_{l \in \mathbb{Z}}|\hat{\varphi}(\omega+2 l \pi)|^{2}\right)\left(\sum_{l \in \mathbb{Z}}|\hat{\varphi}(\omega+2 l \pi)|^{2}\right) \geqslant 1 .
$$

Consequently, the lower bounds also hold with $C_{1}=\tilde{C}_{2}^{-1}$ and $\tilde{C}_{1}=C_{2}^{-1}$. Similarly, it is possible to show that $\{\psi(x-k)\}_{k \in \mathbb{Z}}$ and $\{\tilde{\psi}(x-k)\}_{k \in \mathbb{Z}}$ are Riesz bases for the subspaces they generate.

The stability of the translates at one fixed scale is thus not a problem, provided that $\varphi_{n}$ and $\tilde{\varphi}_{n}$ converge to $\varphi$ and $\tilde{\varphi}$ in $L^{2}(\mathbb{R})$. The real problem is the global stability of the families $\left\{\psi_{k}^{j}\right\}_{j, k \in \mathbb{Z}}$ and $\left\{\tilde{\psi}_{k}^{j}\right\}_{j, k \in \mathbb{Z}}$. It is conceivable that, when the different scales stack up, combining the conditioning constants from all the scales leads to constants which blow up to zero and infinity on each side.

The question of global stability can be reduced to the existence of two upperframe bounds $B, \widetilde{B}>0$ such that, for all $f$ in $L^{2}(\mathbb{R})$,

$$
\begin{aligned}
& \sum_{j, k \in \mathbb{Z}}\left|\left\langle f \mid \psi_{k}^{j}\right\rangle\right|^{2} \leqslant B\|f\|^{2}, \\
& \sum_{j, k \in \mathbb{Z}}\left|\left\langle f \mid \tilde{\psi}_{k}^{j}\right\rangle\right|^{2} \leqslant \tilde{B}\|f\|^{2} .
\end{aligned}
$$

Indeed, if (2.26) - (2.27) hold, then we can use (2.9) and the Cauchy-Schwarz inequality to obtain

$$
\begin{aligned}
\|f\|^{2} & =\lim _{J \rightarrow+\infty} \sum_{j=-J}^{J} \sum_{k \in \mathbb{Z}}\left\langle f \mid \tilde{\psi}_{k}^{j}\right\rangle\left\langle\overline{f \mid \psi_{k}^{j}}\right\rangle \\
& \leqslant\left(\sum_{j, k \in \mathbb{Z}}\left|\left\langle f \mid \tilde{\psi}_{k}^{j}\right\rangle\right|^{2}\right)^{1 / 2}\left(\sum_{j, k \in \mathbb{Z}}\left|\left\langle f \mid \psi_{k}^{j}\right\rangle\right|^{2}\right)^{1 / 2} \\
& \leqslant(\tilde{B})^{1 / 2}\|f\|\left(\sum_{j, k \in \mathbb{Z}}\left|\left\langle f \mid \psi_{k}^{j}\right\rangle\right|^{2}\right)^{1 / 2}
\end{aligned}
$$

so that the lower-frame inequalities are also satisfied with the constants $A=\widetilde{B}^{-1}$ and $\tilde{A}=B^{-1}$.

The biorthogonality and the stability of the families $\left\{\psi_{k}^{j}\right\}_{j, k \in \mathbb{Z}}$ and $\left\{\tilde{\psi}_{k}^{j}\right\}_{j, k \in \mathbb{Z}}$ seem to be two different problems, and in particular the global stability seems to require stronger assumptions than the duality relations (2.18). 
We shall see that, contrary to this intuition, the necessary and sufficient conditions on the dual filters $m_{0}$ and $\tilde{m}_{0}$ that ensure (2.18) also imply (2.26)-(2.27). We now introduce the tools which will be appropriate to state these conditions.

3. Transition operators. Let $m_{0}(\omega)$ be a continuous, $2 \pi$-periodic function such that $m_{0}(0)=1$ and $m_{0}(\pi)=0$. The transition operator $T_{0}$ associated with $m_{0}$ acts on continuous, $2 \pi$-periodic functions in the following way:

$$
T_{0} f(\omega)=\left|m_{0}\left(\frac{\omega}{2}\right)\right|^{2} f\left(\frac{\omega}{2}\right)+\left|m_{0}\left(\frac{\omega}{2}+\pi\right)\right|^{2} f\left(\frac{\omega}{2}+\pi\right)
$$

This operator appears in the works of W. Lawton [La] and J.P. Conze and A. Raugi [CR] for the study of orthonormal wavelets. It can also be used to estimate the regularity (in the Hölder or Sobolev sense) of the scaling function associated to $m_{0}$ (see [E], [Vi], [CD], [CDM]).

Here, we shall need two lemmas that give the basic properties of $T_{0}$.

LEMMA 3.1. Suppose that $m_{0}(\omega)=\sum_{k=0}^{N} h_{k} e^{i k \omega}$ is a trigonometric polynomial and let $T_{0}$ be its associated transition operator. Then the $(2 N+1)$-dimensional space

$$
E_{N}=\left\{\sum_{k=-N}^{N} c_{k} e^{i k \omega} ;\left(c_{-N}, \ldots, c_{N}\right) \in \mathbb{C}^{2 N+1}\right\}
$$

and its subspace

$$
F_{N}=\left\{\sum_{k=-N}^{N} c_{k} e^{i k \omega} ; \sum_{k=-N}^{N} c_{k}=0\right\}
$$

are both stable under the action of $T_{0}$.

Proof. From the definition of $T_{0}$ it is clear that, if $\left|m_{0}\right|^{2}$ and $f$ are two elements of $E_{N}$, then $T_{0} f$ is also in $E_{N}$. A trigonometric polynomial $f$ is in $F_{N}$ if and only if $f \in E_{N}$ and $f(0)=0$. Consequently, if $f$ is in $F_{N}$, we have

$$
T f(0)=\left|m_{0}(0)\right|^{2} f(0)+\left|m_{0}(\pi)\right|^{2} f(\pi)=0,
$$

which proves that $F_{N}$ is stable under the action of $T_{0}$.

Remark. If we consider the Fourier expansion

$$
\left|m_{0}(\omega)\right|^{2}=\sum_{k=-N}^{N} H_{k} e^{i k \omega}
$$


then the matrix of $T_{0}$ restricted to $E_{N}$ is given by

$$
M_{0}=\left(2 H_{i-2 j}\right)_{i, j=-N, \ldots, N}=2\left[\begin{array}{ccccc}
H_{N} & 0 & \cdots & \cdots & 0 \\
H_{N-2} & H_{N-1} & H_{N} & & \vdots \\
\vdots & \vdots & \vdots & & \vdots \\
\vdots & \vdots & \vdots & & 0 \\
H_{-N} & H_{-N+1} & \vdots & & H_{N} \\
0 & 0 & H_{-N} & & H_{N-2} \\
\vdots & \vdots & \vdots & & \vdots \\
\vdots & \vdots & \vdots & & \vdots \\
0 & 0 & \cdots & \cdots & H_{-N}
\end{array}\right]
$$

Since $\left|m_{0}(0)\right|^{2}=\sum_{k=-N}^{N} H_{k}=1$ and $\left|m_{0}(\pi)\right|^{2}=\sum_{k=-N}^{N}(-1)^{k} H_{k}=0$, it follows that

$$
\sum_{k} H_{2 k}=\sum_{k} H_{2 k+1}=\frac{1}{2}
$$

and thus the row vector $\mu=(1, \ldots, 1)$ satisfies

$$
\mu T_{0}=\mu
$$

This is another proof of the fact that $F_{N}$ is stable since $F_{N}=(\mathbb{C} \mu)^{\perp}$. It also shows that 1 is an eigenvalue of $T_{0}$ and that, if this eigenvalue is not degenerate, then it does not belong to the spectrum of $T_{0}$ restricted to $F_{N}$.

The second lemma links iterates of the operator $T_{0}$ with the approximating sequence $\varphi_{n}$, constructed in the previous section, which we would like to converge to $\varphi$ in $L^{2}(\mathbb{R})$.

LEMMA 3.2. For any $2 \pi$-periodic, continuous function $f$, we have

$$
\int_{-\pi}^{\pi} T_{0}^{n} f(\omega) d \omega=\int_{-2^{n} \pi}^{2^{n} \pi} f\left(2^{-n} \omega\right) \prod_{k=1}^{n}\left|m_{0}\left(2^{-k} \omega\right)\right|^{2} d \omega=\int_{\mathbb{R}} f\left(2^{-n} \omega\right)\left|\hat{\varphi}_{n}(\omega)\right|^{2} d \omega .
$$

Proof. We prove it by induction. (3.9) is trivial for $n=0$, and if it is satisfied for some $n \geqslant 0$, then

$$
\begin{aligned}
\int_{-\pi}^{\pi} T_{0}^{n+1} f(\omega) d \omega & =\int_{-\pi}^{\pi} T_{0}^{n}\left(T_{0} f\right)(\omega) d \omega \\
& =\int_{-2^{n} \pi}^{2^{n} \pi} T_{0} f\left(2^{-n} \omega\right) \prod_{k=1}^{n}\left|m_{0}\left(2^{-k} \omega\right)\right|^{2} d \omega
\end{aligned}
$$




$$
\begin{aligned}
= & \int_{-2^{n} \pi}^{2^{n} \pi}\left[f\left(2^{-n-1} \omega\right)\left|m_{0}\left(2^{-n-1} \omega\right)\right|^{2}\right. \\
& \left.+f\left(2^{-n-1} \omega+\pi\right)\left|m_{0}\left(2^{-n-1} \omega+\pi\right)\right|^{2}\right] \prod_{k=1}^{n}\left|m_{0}\left(2^{-k} \omega\right)\right|^{2} d \omega \\
= & \int_{-2^{n+1} \pi}^{2^{n+1} \pi} f\left(2^{-n-1} \omega\right) \prod_{k=1}^{n+1}\left|m_{0}\left(2^{-k} \omega\right)\right|^{2} d \omega .
\end{aligned}
$$

We are now ready to state a first result which gives a sufficient condition for the convergence of $\varphi_{n}$ to $\varphi$ (and similarly $\tilde{\varphi}_{n}$ to $\left.\tilde{\varphi}\right)$ in $L^{2}(\mathbb{R})$. This condition is related to the spectrum of $T_{0}$ restricted to $F_{N}$.

THEOREM 3.3. Let $\lambda$ be the largest eigenvalue of $T_{0}$ restricted to $F_{N}$. If $|\lambda|<1$, then

- $\varphi_{n}$ converges to $\varphi$ in $L^{2}(\mathbb{R})$ and

- $\varphi$ belongs to the Besov space $B_{2}^{s, \infty}(\mathbb{R})$ for all $s<\frac{-\log |\lambda|}{2 \log 2}$.

Proof. Let us define the trigonometric polynomial $c(\omega)$ by

$$
c(\omega)=1-\cos \omega .
$$

It is clear that $c(\omega)$ is in $F_{N}$. (We have supposed $N \geqslant 1$.)

Applying Lemma 3.2, we find that for any $\rho>|\lambda|$

$$
\begin{aligned}
\int\left|\hat{\varphi}_{n}(\omega)\right|^{2} c\left(2^{-n} \omega\right) d \omega & =\int_{-\pi}^{\pi} T_{0}^{n} c(\omega) d \omega \\
& \leqslant \sqrt{2 \pi}\left\|T_{0}^{n} c\right\|_{L^{2}} \\
& \leqslant C \rho^{n} .
\end{aligned}
$$

This leads to an estimate for the $L^{2}$ norm of the Littlewood-Paley blocks of $\varphi$. Since this is an $L^{2}$ estimate, we can define here the dyadic blocks in the Littlewood-Paley decomposition by a simple "cutoff" in the frequency variable:

$$
\hat{\Delta}_{n}(\varphi)(\omega)=\hat{\varphi}(\omega)\left[\chi_{\left[-2^{n} \pi,-2^{n-1} \pi\right]}(\omega)+\chi_{\left[2^{n-1} \pi, 2^{n} \pi\right]}(\omega)\right]
$$

We know that, when $|\omega| \leqslant 2^{n} \pi$, we have

$$
|\hat{\varphi}(\omega)|=\left|\hat{\varphi}_{n}(\omega) \hat{\varphi}\left(2^{-n} \omega\right)\right| \leqslant\left|\hat{\varphi}_{n}(\omega)\right| \max _{|\omega| \leqslant \pi}(|\hat{\varphi}(\omega)|)
$$

We now use the fact that $c(\omega)$ is positive and $c(\omega) \geqslant 1$ when $\pi / 2 \leqslant|\omega| \leqslant \pi$; com- 
bined with the previous statements, this leads to

$$
\begin{aligned}
\left\|\hat{\Delta}_{n}(\varphi)\right\|_{L^{2}}^{2} & =\frac{1}{2 \pi} \int_{2^{n-1} \pi \leqslant|\omega| \leqslant 2^{n} \pi}|\hat{\varphi}(\omega)|^{2} d \omega \\
& \leqslant \frac{1}{2 \pi} \max _{|\omega| \leqslant \pi}\left(|\hat{\varphi}(\omega)|^{2}\right) \int_{2^{n-1} \pi \leqslant|\omega| \leqslant 2^{n \pi}}\left|\hat{\varphi}_{n}(\omega)\right|^{2} d \omega \\
& \leqslant \frac{1}{2 \pi} \max _{|\omega| \leqslant \pi}\left(|\hat{\varphi}(\omega)|^{2}\right) \int_{-2^{n^{n}}}^{2^{n} \pi}\left|\hat{\varphi}_{n}(\omega)\right|^{2} c\left(2^{-n} \omega\right) d \omega \\
& \leqslant C \rho^{n}
\end{aligned}
$$

for all $n \geqslant 0$. If we write $s=-(\log \rho) /(2 \log 2)$, we get

$$
\left\|\Delta_{n}(\varphi)\right\|_{L^{2}} \leqslant C 2^{-n s}
$$

and since $\hat{\varphi}(\omega)$ is bounded (and thus square integrable) on $[-\pi, \pi]$, this means precisely that $\varphi$ belongs to the Besov space $B_{2}^{s, \infty}(\mathbb{R})$ for all $s<-(\log |\lambda|) /(2 \log 2)$. Since $|\lambda|<1$, this also implies that $\varphi$ is in $L^{2}(\mathbb{R})$; the geometric decay of the $\left\|\Delta_{n}(\varphi)\right\|_{L^{2}}$ will be crucial to our final result.

We now prove the $L^{2}$ convergence of the sequence $\varphi_{n}$ to $\varphi$. Since $\hat{\varphi}(0)=1$ and $\hat{\varphi}(\omega)$ is regular, there exists an $\alpha$ in $] 0, \pi]$ such that

$$
|\omega| \leqslant \alpha \Rightarrow|\hat{\varphi}(\omega)| \geqslant C>0 \text {. }
$$

Let us divide $\varphi_{n}$ in two parts: $\varphi_{n}=\varphi_{n}^{1}+\varphi_{n}^{2}$ with

and

$$
\hat{\varphi}_{n}^{1}(\omega)=\hat{\varphi}_{n}(\omega) \chi_{\left[-2^{\left.n_{\alpha}, 2^{n} \alpha\right]}\right.}(\omega)
$$

$$
\hat{\varphi}_{n}^{2}(\omega)=\hat{\varphi}_{n}(\omega)\left(\chi_{\left[-2^{\left.n_{\pi}, 2^{n} \pi\right]}\right.}(\omega)-\chi_{\left[-2^{\left.n_{\alpha}, 2^{n} \alpha\right]}\right.}(\omega)\right)
$$

Clearly, $\hat{\varphi}_{n}^{1}(\omega)$ converges pointwise to $\hat{\varphi}(\omega)$, and by (3.14)

$$
\left|\hat{\varphi}_{n}^{1}(\omega)\right| \leqslant \frac{|\hat{\varphi}(\omega)|}{C}
$$

which implies, by Lebesgue's theorem, the $L^{2}$ convergence of $\varphi_{n}^{1}$ to $\varphi$. We also have

$$
\begin{aligned}
\int\left|\hat{\varphi}_{n}^{2}(\omega)\right|^{2} d \omega & =\int_{2^{n_{\alpha} \leqslant|\omega| \leqslant 2^{n_{\pi}}}}\left|\hat{\varphi}_{n}(\omega)\right|^{2} d \omega \\
& \leqslant \frac{1}{C(\alpha)} \int\left|\hat{\varphi}_{n}(\omega)\right|^{2} c\left(2^{-n} \omega\right) d \omega \\
& \leqslant C \rho^{n}
\end{aligned}
$$


for any $\rho>|\lambda|$, and thus by taking $\rho<1, \varphi_{n}^{2}$ tends to zero in $L^{2}$. Consequently, $\varphi$ is also the $L^{2}$ limit of $\varphi_{n}$.

We have seen how the spectral properties of the transition operators provide information on the square-integrability of the scaling functions and on the $L^{2}$ convergence of the truncated products. We shall prove some converse results in the next section. Provided that the integer translates of the scaling function generate a Riesz basis, we shall see that the spectrum of the associated transition operator has necessarily the properties required in Theorem 3.3 .

4. Riesz bases of translates. In this section we shall examine the consequences of the assumption " $\{\varphi(n-k)\}_{k \in \mathbb{Z}}$ is a Riesz basis" on the transition operator $T_{0}$ associated with $m_{0}$.

As we mentioned in Section 2, this property is equivalent to the existence of two positive constants $C_{2} \geqslant C_{1}>0$ such that

$$
0<C_{1} \leqslant \sum_{l \in \mathbb{Z}}|\hat{\varphi}(\omega+2 l \pi)|^{2} \leqslant C_{2}
$$

uniformly in $\omega$.

It is a well-known fact (see [Me]) that such a family can be orthonormalized into a family of the same type $\{\phi(x-k)\}_{k \in \mathbb{Z}}$, where $\phi$ is defined by

$$
\hat{\phi}(\omega)=\hat{\varphi}(\omega)\left(\sum_{l \in \mathbb{Z}}|\hat{\varphi}(\omega+2 l \pi)|^{2}\right)^{-1 / 2} .
$$

We have indeed

$$
\phi(x)=\sum_{k \in \mathbb{Z}} \alpha_{k} \varphi(x-k)
$$

where $\alpha_{k}$ is the coefficient of $e^{-i k \omega}$ in the Fourier decomposition of the $2 \pi$-periodic function $\left(\sum_{l \in \mathbb{Z}}|\hat{\varphi}(\omega+2 l \pi)|^{2}\right)^{-1 / 2}=(m(\omega))^{-1 / 2}$. Note that $m(\omega)=\sum_{l \in \mathbb{Z}}|\hat{\varphi}(\omega+2 l \pi)|^{2}$ is a trigonometric polynomial (because its coefficients are given by the inner products $\langle\varphi(x) \mid \varphi(x-k)\rangle)$ and that it does not vanish because of $(4.1)$. Thus $(m(\omega))^{-1 / 2}$ is a smooth $2 \pi$-periodic function, and the $\alpha_{k}$ have rapid decay at infinity.

The definition (4.2) implies

$$
\sum_{l \in \mathbb{Z}}|\hat{\phi}(\omega+2 l \pi)|^{2}=1
$$

which expresses the orthogonality of the translates

$$
\langle\phi(x) \mid \phi(x-k)\rangle=\frac{1}{2 \pi} \int_{-\pi}^{\pi} \sum_{l \in \mathbb{Z}}|\hat{\phi}(\omega+2 l \pi)|^{2} e^{-i k \omega} d \omega=\delta_{0, k} .
$$


If the scaling function $\varphi$ is generated from a trigonometric polynomial $m_{0}(\omega)$, i.e., $\hat{\varphi}(\omega)=\prod_{k=1}^{+\infty} m_{0}\left(2^{-k} \omega\right)$, we also have

$$
\hat{\phi}(\omega)=\prod_{k=1}^{+\infty} p_{0}\left(2^{-k} \omega\right)
$$

with

$$
p_{0}(\omega)=m_{0}(\omega)\left(\frac{\sum_{l \in \mathbb{Z}}|\hat{\varphi}(2 \omega+2 l \pi)|^{2}}{\sum_{l \in \mathbb{Z}}|\hat{\varphi}(\omega+2 l \pi)|^{2}}\right)^{-1 / 2}=m_{0}(\omega)\left[\frac{m(2 \omega)}{m(\omega)}\right]^{-1 / 2}
$$

In general, $p_{0}(\omega)$ is not a trigonometric polynomial (since $\phi$ is not compactly supported), but it is a smooth function. It satisfies the $\mathrm{CQF}$ equation

$$
\left|p_{0}(\omega)\right|^{2}+\left|p_{0}(\omega+\pi)\right|^{2}=1
$$

characteristic of the orthonormal case.

Let $T_{0}$ and $R_{0}$ be the transition operators respectively associated to $m_{0}(\omega)$ and $p_{0}(\omega)$. The following lemma relates their spectral properties.

Lemma 4.1. Suppose that $\lambda$ is an eigenvalue of $T_{0}$ restricted to $E_{N}$ and let $f_{\lambda}(\omega)$ be a trigonometric polynomial in $E_{N}$ such that $T_{0} f_{\lambda}=\lambda f_{\lambda}$. Then $\lambda$ is also an eigenvalue of $R_{0}$, and the corresponding eigenfunction $g_{\lambda}$ is given by

$$
g_{\lambda}(\omega)=\frac{f_{\lambda}(\omega)}{m(\omega)}
$$

Proof. It suffices to compute

$$
\begin{aligned}
R_{0} g_{\lambda}(\omega) & =\left|p_{0}\left(\frac{\omega}{2}\right)\right|^{2} g_{\lambda}\left(\frac{\omega}{2}\right)+\left|p_{0}\left(\frac{\omega}{2}+\pi\right)\right|^{2} g_{\lambda}\left(\frac{\omega}{2}+\pi\right) \\
& =\left|m_{0}\left(\frac{\omega}{2}\right)\right|^{2} \frac{f_{\lambda}\left(\frac{\omega}{2}\right)}{m(\omega)}+\left|m_{0}\left(\frac{\omega}{2}+\pi\right)\right|^{2} \frac{f_{\lambda}\left(\frac{\omega}{2}+\pi\right)}{m(\omega)} \\
& =\frac{T_{0} f_{\lambda}(\omega)}{m(\omega)}=\lambda g_{\lambda}(\omega) .
\end{aligned}
$$

Note that $g_{\lambda}(\omega)$ is no longer a trigonometric polynomial but is the ratio of two trigonometric polynomials. We shall use this property in the main theorem of this section which will give some significant information on the eigenvalue of $T_{0}$, using the corresponding eigenfunctions of $R_{0}$. We start with a preliminary result on the filters associated to Riesz bases of translates. 
LEMMA 4.2. If $\{\varphi(x-k)\}_{k \in \mathbb{Z}}$ is a Riesz basis, then

- for all $\omega$ in $[-\pi, \pi] \backslash\{0\}, \lim _{n \rightarrow+\infty} \prod_{k=0}^{n} m_{0}\left(2^{k} \omega\right)=0$ and

- for all $\omega$ in $[-\pi, \pi] \backslash\{0\}, \lim _{n \rightarrow+\infty} \prod_{k=0}^{n} p_{0}\left(2^{k} \omega\right)=0$

(with $p_{0}(\omega)$ defined as in (4.7)).

Proof. Since $\varphi(x)$ is compactly supported and square-integrable, it is in $L^{1}(\mathbb{R})$ by the Cauchy-Schwarz inequality. Consequently,

$$
\lim _{|\omega| \rightarrow+\infty} \hat{\varphi}(\omega)=0
$$

Let $\omega_{0}$ be in $[-\pi, \pi] \backslash\{0\}$. Since $\sum_{l \in \mathbb{Z}}|\hat{\varphi}(\omega+2 l \pi)|^{2}>C_{1}>0$ by (4.1), there exists an integer $l$ such that $\hat{\varphi}\left(\omega_{0}+2 l \pi\right) \neq 0$. Since $\omega_{l}=\omega_{0}+2 l \pi$ is different from zero, then necessarily the expression

$$
\hat{\varphi}\left(2^{n} \omega_{l}\right)=\prod_{k=0}^{n-1} m_{0}\left(2^{k} \omega_{l}\right) \hat{\varphi}\left(\omega_{l}\right)=\prod_{k=0}^{n-1} m_{0}\left(2^{k} \omega_{0}\right) \hat{\varphi}\left(\omega_{l}\right)
$$

must converge to zero when $n$ goes to $+\infty$. This is possible only if $\prod_{k=0}^{n} m_{0}\left(2^{k} \omega_{0}\right)$ tends to zero.

The same result can be proved for $p_{0}$ since we have

$$
\begin{aligned}
\left|\prod_{k=0}^{n} p_{0}\left(2^{k} \omega\right)\right| & =\left|\prod_{k=0}^{n} m_{0}\left(2^{k} \omega\right)\right|\left(\frac{m\left(2^{n+1} \omega\right)}{m(\omega)}\right)^{-1 / 2} \\
& \leqslant\left|\prod_{k=0}^{n} m_{0}\left(2^{k} \omega\right)\right|\left(\frac{\max (m(\omega))}{\min (m(\omega))}\right)^{1 / 2} \\
& \leqslant\left|\prod_{k=0}^{n} m_{0}\left(2^{k} \omega\right)\right| \sqrt{C_{2} / C_{1}} .
\end{aligned}
$$

Like Lemma 4.1, Lemma 4.2 will be useful in the sense that we shall work on $p_{0}$ and $R_{0}$ rather than $m_{0}$ and $T_{0}$ to prove the main result of this section.

THEOREM 4.3. If $\{\varphi(x-k)\}_{k \in \mathbb{Z}}$ is a Riesz basis, then the largest eigenvalue $\lambda$ of $T_{0}$ restricted to $F_{N}$ satisfies $|\lambda|<1$.

Proof. We shall first prove that $|\lambda|$ cannot be strictly superior to 1 .

Indeed, let $g_{\lambda}(\omega)$ be the eigenfunction of $R_{0}$ associated to $\lambda$ as in Lemma 4.1 and choose $\omega_{0}$ such that

$$
\left|g_{\lambda}\left(\omega_{0}\right)\right|=\max _{\omega \in \mathbb{R}}\left|g_{\lambda}(\omega)\right|>0
$$


We thus have

$$
\begin{aligned}
\left|\lambda g_{\lambda}\left(\omega_{0}\right)\right| & =\left|R_{0} g_{\lambda}\left(\omega_{0}\right)\right| \\
& =\left.|| p_{0}\left(\frac{\omega_{0}}{2}\right)\right|^{2} g_{\lambda}\left(\frac{\omega_{0}}{2}\right)+\left|p_{0}\left(\frac{\omega_{0}}{2}+\pi\right)\right|^{2} g_{\lambda}\left(\frac{\omega_{0}}{2}+\pi\right) \mid \\
& \leqslant\left|p_{0}\left(\frac{\omega_{0}}{2}\right)\right|^{2}\left|g_{\lambda}\left(\frac{\omega_{0}}{2}\right)\right|+\left|p_{0}\left(\frac{\omega_{0}}{2}+\pi\right)\right|^{2}\left|g_{\lambda}\left(\frac{\omega_{0}}{2}+\pi\right)\right| \\
& \leqslant\left(\left|p_{0}\left(\frac{\omega_{0}}{2}\right)\right|^{2}+\left|p_{0}\left(\frac{\omega_{0}}{2}+\pi\right)\right|^{2}\right)\left|g_{\lambda}\left(\omega_{0}\right)\right|=\left|g_{\lambda}\left(\omega_{0}\right)\right|,
\end{aligned}
$$

and this implies

$$
|\lambda| \leqslant 1
$$

We still have to prove that $\lambda$ does not belong to the unit circle.

Suppose that this was the case (i.e., $|\lambda|=1)$ and let $g_{\lambda}(\omega)$ be the associated eigenfunction of $R_{0}$. We have

$$
g_{\lambda}(0)=\frac{f_{\lambda}(0)}{m(0)}=0
$$

because $f_{\lambda}(\omega)$ is in $F_{N}$. This shows that $\left|g_{\lambda}(\omega)\right|$ is not a constant function. Since $g_{\lambda}$ is the ratio of two trigonometric polynomials, it also implies that any value of $\left|g_{\lambda}\right|$ is attained only at a finite number of points $\omega$ in $[-\pi, \pi]$. Indeed, the equation

$$
\left|g_{\lambda}(\omega)\right|=C
$$

leads to

$$
\left|f_{\lambda}(\omega)\right|^{2}-C|m(\omega)|^{2}=0
$$

which is satisfied either for all $\omega$ in $[-\pi, \pi]$ or for only a finite set since $\left|f_{\lambda}\right|^{2}-C|m|^{2}$ is a trigonometric polynomial.

A direct consequence is that $\left|g_{\lambda}(\omega)\right|$ has a finite number of maxima. Let us choose $\omega_{0}$ in $[-\pi, \pi]$ such that $\left|g_{\lambda}\left(\omega_{0}\right)\right|=\max \left|g_{\lambda}(\omega)\right|$. Clearly, we have

$$
\begin{aligned}
\left|g_{\lambda}\left(\omega_{0}\right)\right| & =\left|\lambda g_{\lambda}\left(\omega_{0}\right)\right| \leqslant\left|p_{0}\left(\frac{\omega_{0}}{2}\right)\right|^{2}\left|g_{\lambda}\left(\frac{\omega_{0}}{2}\right)\right|+\left|p_{0}\left(\frac{\omega_{0}}{2}+\pi\right)\right|^{2}\left|g_{\lambda}\left(\frac{\omega_{0}}{2}+\pi\right)\right| \\
& \leqslant \max \left(\left|g_{\lambda}\left(\frac{\omega_{0}}{2}\right)\right|,\left|g_{\lambda}\left(\frac{\omega_{0}}{2}+\pi\right)\right|\right) .
\end{aligned}
$$


This implies that at least one element of the pair $\left\{\frac{\omega_{0}}{2}, \frac{\omega_{0}}{2}+\pi\right\}$ corresponds to a maximum of $\left|g_{\lambda}\right|$ and that the inequalities in the formula above are in fact equalities.

We shall use the notations

$$
S_{0}(\omega)=\frac{\omega}{2} \quad \text { and } \quad S_{1}(\omega)=\frac{\omega}{2}+\pi
$$

Clearly, $S_{0}$ and $S_{1}$ are the two possible preimages of $\omega$ by the transformation $\omega \mapsto 2 \omega$ modulo $2 \pi$. If we use the binary expansion of $\omega / 2 \pi$, for $\omega$ in $[-\pi, \pi]$,

$$
\frac{\omega}{2 \pi}=\sum_{j=1}^{+\infty} \alpha_{j} 2^{-j} \quad\left(\alpha_{j} \in\{0,1\}\right),
$$

then the action of $S_{0}$ consists in adding a " 0 " in the beginning of the sequence $\left\{\alpha_{j}\right\}_{j \geqslant 1}$, whereas the action of $S_{1}$ adds a " 1 ".

Since at least one among $S_{0}\left(\omega_{0}\right)$ and $S_{1}\left(\omega_{0}\right)$ corresponds to a maximum of $\left|g_{\lambda}\right|$, we can iterate this process and build a sequence

$$
\omega_{j}=S_{\varepsilon_{j}}\left(\omega_{j-1}\right) \quad \varepsilon_{j}=0 \text { or } 1
$$

such that $\left|g_{\lambda}\left(\omega_{j}\right)\right|=\max _{\omega}\left|g_{\lambda}(\omega)\right|$, but this sequence can only reach a finite set of different values. Consequently, the binary expansion of $\omega_{0} / 2 \pi$ given by (4.18) must be periodic, and so must the sequence $\left\{\omega_{j}\right\}_{j \geqslant 1}$. This implies that for a given $j$ we can only apply one of the two transformations $S_{0}$ and $S_{1}$ on $\omega_{j}$ to obtain a new maximum of $\left|g_{\lambda}\right|$ at $\omega_{j+1}=S_{\varepsilon_{j+1}} \omega_{j}$, where $\varepsilon_{j}$ is fixed by the periodic structure of the binary expansion (4.18). The other preimage of $\omega_{j}$, i.e., $\omega_{j+1}+\pi$ (modulo $2 \pi$ ) will not be a maximum of $\left|g_{\lambda}\right|$. But we have for all $j \geqslant 0$

$$
\left|g_{\lambda}\left(\omega_{j+1}\right)\right|=\left|p_{0}\left(\omega_{j}\right)\right|^{2}\left|g_{\lambda}\left(\omega_{j}\right)\right|+\left|p_{0}\left(\omega_{j}+\pi\right)\right|^{2}\left|g_{\lambda}\left(\omega_{j}+\pi\right)\right|
$$

with $\left|p_{0}\left(\omega_{j}\right)\right|^{2}+\left|p_{0}\left(\omega_{j}+\pi\right)\right|^{2}=1$, so that necessarily

$$
\left|p_{0}\left(\omega_{j}\right)\right|=1 \quad \text { and } \quad p_{0}\left(\omega_{j}+\pi\right)=0 .
$$

We now note that the sequence $\left\{2^{k} \omega_{0}\right\}_{k \geqslant 0}$ is exactly the sequence $\left\{\omega_{j}\right\}_{j \geqslant 0}$ modulo $2 \pi$ but described in the reverse order. (We could write $2^{k} \omega_{0}=\omega_{-k}$ modulo $2 \pi$.) This leads to

$$
\prod_{k=0}^{n}\left|p_{0}\left(2^{k} \omega_{0}\right)\right|=1 \quad \text { for all } n \geqslant 0
$$

Since $\omega_{0}$ is different from zero $\left(g_{\lambda}(0)=0\right)$, this contradicts the result of Lemma 4.2, and we conclude that the spectral radius of $T_{0}$ restricted to $F_{N}$ has to satisfy $|\lambda|<1$. 
We see that the conclusion of Theorem 4.3 is exactly the hypothesis required in Theorem 3.3. In the next section we shall thus combine these two results to obtain the exact characterization of the dual filters that lead to biorthogonal Riesz bases of compactly supported wavelets.

5. Necessary and sufficient conditions for biorthogonality and stability. The goal of this section is to give a proof and a typical application of the following theorem.

THEOREM 5.1. A pair of dual filters $\left\{m_{0}(\omega), \tilde{m}_{0}(\omega)\right\}$ generates biorthogonal Riesz bases of compactly supported wavelets if and only if the associated transition operators $T_{0}$ and $\widetilde{T}_{0}$ satisfy

$$
\left|\rho\left(T_{0}\right)\right|<1 \quad \text { and } \quad\left|\rho\left(\tilde{T}_{0}\right)\right|<1
$$

where $\rho\left(T_{0}\right)\left(\right.$ resp. $\left.\rho\left(\tilde{T}_{0}\right)\right)$ is the spectral radius of $T_{0}\left(\right.$ resp. $\left.\tilde{T}_{0}\right)$ restricted to $F_{N}\left(\right.$ resp. $\left.\tilde{F}_{N}\right)$.

Proof. We first prove the necessity of this condition: Suppose that $m_{0}$ and $\tilde{m}_{0}$ lead to a pair of biorthogonal Riesz bases of wavelets. Then Proposition 2.1 tells us that we have

$$
\langle\varphi(x-k) \mid \tilde{\varphi}(x-l)\rangle=\delta_{k, l}
$$

and we have shown in Section 2 that this implies that $\{\varphi(x-k)\}_{k \in \mathbb{Z}}$ and $\{\tilde{\varphi}(x-k)\}_{k \in \mathbb{Z}}$ are both Riesz bases for the subspaces that they generate. The condition (5.1) follows by Theorem 4.3.

We now prove sufficiency. By Theorem 3.3, the condition (5.1) implies the $L^{2}$ convergence of the sequences $\varphi_{n}$ and $\tilde{\varphi}_{n}$ to $\varphi$ and $\tilde{\varphi}$. By Proposition 2.1, we have for any $f$ in $L^{2}(\mathbb{R})$

$$
f=\lim _{J \rightarrow+\infty} \sum_{j=-J}^{J} \sum_{k \in \mathbb{Z}}\left\langle f \mid \tilde{\psi}_{k}^{j}\right\rangle \psi_{k}^{j},
$$

and for all $j, j^{\prime}, k$, and $k^{\prime}$ in $\mathbb{Z}$

$$
\left\langle\psi_{k}^{j} \mid \tilde{\psi}_{k^{\prime}}^{j^{\prime}}\right\rangle=\delta_{j, j^{\prime}} \delta_{k, k^{\prime}}
$$

As we mentioned in Section 2, the stability will be achieved if there exist two upper-frame bounds $B$ and $\widetilde{B}$ such that, for all $f$ in $L^{2}(\mathbb{R})$,

$$
\begin{gathered}
\sum_{j, k \in \mathbb{Z}}\left|\left\langle f \mid \psi_{k}^{j}\right\rangle\right|^{2} \leqslant B\|f\|^{2}, \\
\sum_{j, k \in \mathbb{Z}}\left|\left\langle f \mid \tilde{\psi}_{k}^{j}\right\rangle\right|^{2} \leqslant \tilde{B}\|f\|^{2} .
\end{gathered}
$$

To construct $B$ (and $\widetilde{B}$ in a similar way) we use that, by Theorem $3.3, \varphi$ belongs to the Besov space $B_{2}^{s, \infty}(\mathbb{R})$ for all $s<-\left(\log \left|\rho\left(T_{0}\right)\right|\right) / 2 \log 2$. 
We first prove the useful estimates

$$
\text { for all } \varepsilon<\frac{-2 \log \left|\rho\left(T_{0}\right)\right|}{\log 2-\log \left|\rho\left(T_{0}\right)\right|}, \quad \sum_{l \in \mathbb{Z}}|\hat{\varphi}(\omega+2 l \pi)|^{2-\varepsilon}<C(\varepsilon)
$$

and

$$
\text { for all } \varepsilon<\frac{-\log \left|\rho\left(T_{0}\right)\right|}{4 \log 2}, \quad|\hat{\varphi}(\omega)|(1+|\omega|)^{\varepsilon}<C(\varepsilon)
$$

where $C(\varepsilon), C^{\prime}(\varepsilon)$ are constants that do not depend on $\omega$. Since $m_{0}(\pi)=m_{0}(-\pi)=0$, the scaling function satisfies

$$
\hat{\varphi}(2 l \pi)=0 \quad \text { if } l \in \mathbb{Z} \backslash\{0\} .
$$

We have

$$
\begin{aligned}
\sum_{l \in \mathbb{Z}}|\hat{\varphi}(\omega+2 l \pi)|^{2-\varepsilon} & \leqslant \int\left|\frac{d}{d \omega}\left[|\hat{\varphi}|^{2-\varepsilon}\right]\right| d \omega \\
& =\int\left|\frac{d}{d \omega}\left[\left(|\hat{\varphi}|^{2}\right)^{1-(\varepsilon / 2)}\right]\right| d \omega \\
& =\int\left(1-\frac{\varepsilon}{2}\right)\left|\frac{d}{d \omega}\left[|\hat{\varphi}|^{2}\right]\right||\hat{\varphi}|^{-\varepsilon} d \omega \\
& \leqslant(2-\varepsilon) \int\left|\frac{d \hat{\varphi}}{d \omega}\right||\hat{\varphi}(\omega)|^{1-\varepsilon} d \omega \\
& \leqslant C\left(\int\left|\frac{d \hat{\varphi}}{d \omega}\right|^{2} d \omega\right)^{1 / 2}\left(\int|\hat{\varphi}(\omega)|^{2-2 \varepsilon} d \omega\right)^{1 / 2}
\end{aligned}
$$

The first factor is proportional to the $L^{2}$ norm of $x \varphi(x)$, which is finite since $\varphi$ is square integrable and compactly supported.

To evaluate the second factor, we compute the integral of $|\hat{\varphi}(\omega)|^{2-2 \varepsilon}$ on a dyadic ring $2^{n-1} \pi \leqslant|\omega| \leqslant 2 \pi$. By the Hölder inequality and (3.13), we obtain

$$
\begin{aligned}
\int_{2^{n-1} \pi \leqslant|\omega| \leqslant 2^{n} \pi}|\hat{\varphi}(\omega)|^{2-2 \varepsilon} d \omega & \leqslant\left[\int_{2^{n-1} \pi \leqslant|\omega| \leqslant 2^{n} \pi}|\hat{\varphi}(\omega)|^{2} d \omega\right]^{1-\varepsilon}\left(2^{n} \pi\right)^{\varepsilon} \\
& \leqslant C 2^{n \varepsilon-(2-2 \varepsilon) s}
\end{aligned}
$$

for all $s<-\log \left|\rho\left(T_{0}\right)\right| /(2 \log 2)$. 
The second factor will thus be finite and (5.7) will be satisfied if we choose $\varepsilon$ such that $\varepsilon+(2-2 \varepsilon)\left(\log \left|\rho\left(T_{0}\right)\right| / 2 \log 2\right)<0$, i.e., $\varepsilon<-\log \left|\rho\left(T_{0}\right)\right| /\left(\log 2-\log \left|\rho\left(T_{0}\right)\right|\right)$. We apply the same method to prove the estimate (5.8). If $|\omega|$ is in $\left[2^{n-1} \pi, 2^{n} \pi\right]$ for $n \geqslant 1$, we can write

$$
\begin{aligned}
|\hat{\varphi}(\omega)|^{2} & \leqslant \int_{2^{n-1} \pi \leqslant|\omega| \leqslant 2^{n} \pi} \frac{d}{d \omega}\left[|\hat{\varphi}|^{2}\right] d \omega \\
& \leqslant C\left(\int_{\mathbb{R}}\left|\frac{d \hat{\varphi}}{d \omega}\right|^{2} d \omega\right)^{1 / 2}\left(\int_{2^{n-1} \pi \leqslant|\omega| \leqslant 2^{n} \pi}|\hat{\varphi}(\omega)|^{2} d \omega\right)^{1 / 2} \\
& \leqslant C 2^{-n 2 \varepsilon} \leqslant C(1+|\omega|)^{-2 \varepsilon}
\end{aligned}
$$

if $2 \varepsilon<-\log \left|\rho\left(T_{0}\right)\right| / 2 \log 2$.

Note that to show (5.7) and (5.8) we only used estimates of $|\hat{\varphi}|^{2}$ or its integral on dyadic rings $2^{n-1} \pi \leqslant|\omega| \leqslant 2^{n} \pi$ for $n \geqslant 1$. The case $|\omega| \leqslant \pi$ does not cause any problem since $\hat{\varphi}(\omega)$ is a bounded function on $[-\pi, \pi]$.

We now use (5.7) and (5.8) to prove (5.5). Since $\hat{\psi}(\omega)=m_{1}\left(\frac{\omega}{2}\right) \hat{\varphi}\left(\frac{\omega}{2}\right)$ has at least a first-order zero at the origin, we can write, using (5.7) and (5.8),

$$
\sum_{j \in \mathbb{Z}}\left|\hat{\psi}\left(2^{j} \omega\right)\right|^{\varepsilon} \leqslant C_{1}(\varepsilon)
$$

and

$$
\sum_{l \in \mathbb{Z}}|\hat{\psi}(\omega+2 l \pi)|^{2-\varepsilon} \leqslant C_{2}(\varepsilon)
$$

for all $\varepsilon<-\log \left|\rho\left(T_{0}\right)\right| /\left(\log 2-\log \left|\rho\left(T_{0}\right)\right|\right)$.

Using Plancherel's formula and the Poisson summation formula, we can derive for all $f$ in $L^{2}(\mathbb{R})$

$$
\begin{aligned}
\sum_{k \in \mathbb{Z}}\left|\left\langle f \mid \psi_{k}^{j}\right\rangle\right|^{2} & =\frac{1}{2 \pi} \sum_{k \in \mathbb{Z}} 2^{j}\left|\int_{\mathbb{R}} \hat{f}(\omega) \hat{\psi}\left(2^{j} \omega\right) e^{-i 2^{j k} \omega} d \omega\right|^{2} \\
& =\frac{1}{2 \pi} \sum_{k \in \mathbb{Z}} 2^{-j}\left|\int_{\mathbb{R}} \hat{f}\left(2^{-j} \omega\right) \hat{\psi}(\omega) e^{-i k \omega} d \omega\right|^{2} \\
& =2^{-j} \int_{-\pi}^{\pi}\left|\sum_{l \in \mathbb{Z}} \hat{f}\left(2^{-j}(\omega+2 l \pi)\right) \hat{\psi}(\omega+2 l \pi)\right|^{2} d \omega
\end{aligned}
$$




$$
\begin{aligned}
& \leqslant 2^{-j} \int_{-\pi}^{\pi}\left(\sum_{l \in \mathbb{Z}}\left|\hat{f}\left(2^{-j}(\omega+2 l \pi)\right)\right||\hat{\psi}(\omega+2 l \pi)|^{\varepsilon / 2}|\hat{\psi}(\omega+2 l \pi)|^{1-(\varepsilon / 2)}\right)^{2} d \omega \\
& \leqslant 2^{-j} \int_{-\pi}^{\pi}\left(\sum_{l \in \mathbb{Z}}\left|\hat{f}\left(2^{-j}(\omega+2 l \pi)\right)\right|^{2}|\hat{\psi}(\omega+2 l \pi)|^{\varepsilon}\right)\left(\sum_{l \in \mathbb{Z}}|\hat{\psi}(\omega+2 l \pi)|^{2-\varepsilon}\right) d \omega \\
& \leqslant C_{2}(\varepsilon) 2^{-j} \int_{\mathbb{R}}\left|\hat{f}\left(2^{-j} \omega\right)\right|^{2}|\hat{\psi}(\omega)|^{\varepsilon} d \omega \\
& \leqslant C_{2}(\varepsilon) \int_{\mathbb{R}}|\hat{f}(\omega)|^{2}\left|\hat{\psi}\left(2^{j} \omega\right)\right|^{\varepsilon} d \omega .
\end{aligned}
$$

Summing over all the scales $j \in \mathbb{Z}$, we obtain

$$
\begin{aligned}
\sum_{j, k \in \mathbb{Z}}\left|\left\langle f \mid \psi_{k}^{j}\right\rangle\right|^{2} & \leqslant C_{1}(\varepsilon) C_{2}(\varepsilon) \int_{\mathbb{R}}|\hat{f}(\omega)|^{2} d \omega \\
& =2 \pi C_{1}(\varepsilon) C_{2}(\varepsilon)\|f\|^{2}
\end{aligned}
$$

which gives the upper-frame bound $B=2 \pi C_{1}(\varepsilon) C_{2}(\varepsilon)$. Since the same holds for $\tilde{\psi}$, this concludes the proof of the theorem.

We have obtained a complete characterization of the dual filters leading to stable biorthogonal wavelet bases. To illustrate the interest of this result, let us present a typical example where it applies fruitfully. In [CDF] we have constructed biorthogonal bases in the case where $\varphi$ and $\psi$ are spline (i.e., piecewise polynomial) functions. The principle is the following: For a fixed $N$, which will be the degree of our spline wavelets, we define

$$
m_{0}^{N}(\omega)=\left(\frac{1+e^{-i \omega}}{2}\right)^{N+1}
$$

so that the corresponding scaling function $\varphi_{N}$ is the $B$-spline of order $N$, which can also be obtained by convolving $\chi_{[0,1]} N$ times with itself:

$$
\varphi_{N}(x)=(*)^{N+1} \chi_{[0,1]}
$$

To find a dual filter $\tilde{m}_{0}(\omega)$, we use the polynomial

$$
P_{L}(y)=\sum_{j=0}^{L-1}\left[\begin{array}{c}
L-1+j \\
j
\end{array}\right] y^{i}
$$

that solves the Bezout equation

$$
y^{L} P_{L}(1-y)+(1-y)^{L} P_{L}(y)=1
$$


By a change of variable, this formula gives a solution $\tilde{m}_{0}^{N, L}(\omega)$ for all the values of $N$ smaller than $2 L-1$, namely,

$$
\tilde{m}_{0}^{N, L}(\omega)=\left[\frac{1+e^{i \omega}}{2}\right]^{2 L-N-1} P_{L}\left(\sin ^{2}\left(\frac{\omega}{2}\right)\right) e^{-i L \omega}
$$

By taking a large value for $L$, we increase the regularity of $\tilde{\varphi}$. A good choice for this parameter is the smallest value such that $2 L-1$ is superior to $N$ and such that $\tilde{m}_{0}^{N, L}(\omega)$ satisfies the criterion of Theorem 5.1. (It is easy to check that $m_{0}^{N}(\omega)$ does not cause any problem because of the regularity of $\varphi_{N}$.)

The case of quadratic splines $(N=2)$ is particularly interesting. Since $N=2$, we must have $2 L \geqslant 3$, i.e., $L \geqslant 2$. The choice $L=2$ would give

$$
\tilde{m}_{0}^{2,2}(\omega)=\frac{1}{4}\left(-e^{-3 i \omega}+3 e^{-2 i \omega}+3 e^{-i \omega}-1\right)
$$

Note that we have

$$
\left|\tilde{m}_{0}^{2,2}\left(\frac{2 \pi}{3}\right)\right|=\left|\tilde{m}_{0}^{2,2}\left(\frac{4 \pi}{3}\right)\right|=1
$$

and thus for all $n>0$

$$
\left|\hat{\tilde{\varphi}}_{2,2}\left(\frac{2^{n} \pi}{3}\right)\right|=\left|\prod_{k=1}^{n-1} \tilde{m}_{0}^{2,2}\left(\frac{2^{k} \pi}{3}\right)\right|\left|\hat{\tilde{\varphi}}_{2,2}\left(\frac{2 \pi}{3}\right)\right|=\left|\hat{\tilde{\varphi}}_{2,2}\left(\frac{2 \pi}{3}\right)\right| \neq 0 .
$$

This shows that $\tilde{\varphi}_{2,2}$ cannot be in $L^{2}(\mathbb{R})$. Indeed, if it were, it would also be in $L^{1}(\mathbb{R})$ since it is a compactly supported function, and $\left|\hat{\tilde{\varphi}}_{2,2}(\omega)\right|$ should have some decay at infinity. We must thus give up $L=2$.

Let us now consider the choice $L=3$ which leads to

$$
\tilde{m}_{0}^{2,3}(\omega)=\frac{1}{64}\left(3\left(e^{-5 i \omega}+e^{2 i \omega}\right)-9\left(e^{-h i \omega}+e^{i \omega}\right)-7\left(e^{-3 i \omega}+1\right)+45\left(e^{-2 i \omega}+e^{-i \omega}\right)\right)
$$

and

$$
\begin{aligned}
\left|\tilde{m}_{0}^{2,3}(\omega)\right|^{2}= & 2^{-11}(2164+1467 \cos \omega-1482 \cos 2 \omega-491 \cos 3 \omega+396 \cos 4 \omega \\
& +39 \cos 5 \omega-54 \cos 6 \omega+9 \cos 7 \omega)
\end{aligned}
$$

In the basis $(1, \cos \omega, \cos 2 \omega, \cos 3 \omega, \cos 4 \omega, \cos 5 \omega, \cos 6 \omega)$, the matrix of $\tilde{T}_{0}$ can 
be written as

$$
\tilde{M}_{0}=2^{-11}\left[\begin{array}{rrrrrrr}
4328 & 1467 & -1482 & -491 & 396 & 39 & -54 \\
-2964 & 976 & 4724 & 1506 & -1536 & -482 & 396 \\
792 & -452 & -1536 & 1476 & 4328 & 1467 & -1482 \\
-108 & 48 & 396 & -491 & -1482 & 1467 & 4328 \\
0 & 9 & -54 & 39 & 396 & -491 & -1482 \\
0 & 0 & 0 & 9 & -54 & 39 & 396 \\
0 & 0 & 0 & 0 & 0 & 9 & -54
\end{array}\right]
$$

The characteristic polynomial of $\tilde{M}_{0}$ has the form

$$
\left\{\begin{array}{l}
p_{0}(x)=(x-1)\left(x-\frac{1}{4}\right)\left(x-\frac{1}{16}\right)(x-\alpha)(x-\rho)(x-\gamma)(x-\delta) \\
\alpha \simeq .784, \beta \simeq-.447,-.175, \text { and } \delta \simeq-.038
\end{array}\right.
$$

Except for the nondegenerate eigenvalue 1, all the other eigenvalues are inside the unit disk, so that $\tilde{m}_{0}^{2,3}(\omega)$ satisfies the criterion of Theorem 5.1.

Note however that we have $\left|\tilde{m}_{0}^{2,3}\left(\frac{2 \pi}{3}\right)\right|=\frac{23}{32}$, and thus for all $n>0$

$$
\left|\hat{\tilde{\varphi}}_{2,3}\left(\frac{2^{n} \pi}{3}\right)\right|=\left|\prod_{k=1}^{n-1} \tilde{m}_{0}^{2,3}\left(\frac{2^{k} \pi}{3}\right)\right|\left|\hat{\tilde{\varphi}}_{2,3}\left(\frac{2 \pi}{3}\right)\right| \sim C\left(\frac{2^{k} \pi}{3}\right)\left[\log _{2}\left(\frac{23}{32}\right)\right] \text {, }
$$
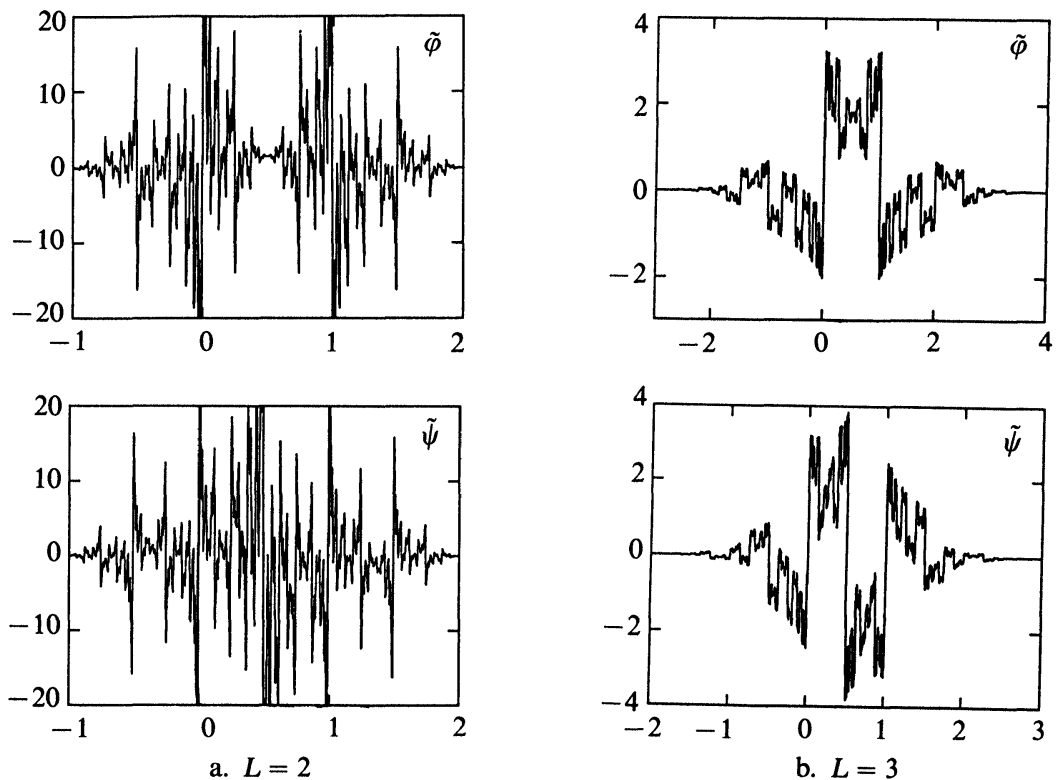

$\tilde{\varphi}$ and $\tilde{\psi}$ are not in $L^{2}(\mathbb{R})$. No stable basis can be derived.

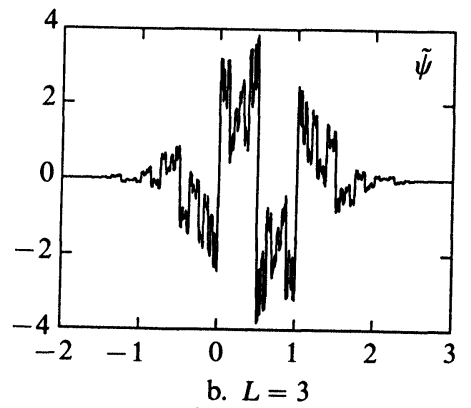

$\tilde{\varphi}$ and $\tilde{\psi}$ are in $L^{2}(\mathbb{R})$, but $\operatorname{Sup}(1+|\omega|)^{1 / 2}|\hat{\varphi}(\omega)|=+\infty$.

Figure 3. Quadratic spline wavelets $(N=2)$ 
and since $\frac{23}{32}>\frac{\sqrt{2}}{2}$, the decay of $\hat{\tilde{\varphi}}_{2,3}(\omega)$ at infinity is weaker than $|\omega|^{-1 / 2}$. It is nevertheless square-integrable because of its "lacunary" aspect: the regions where $\hat{\tilde{\varphi}}_{2,3}(\omega)$ decays badly become less and less dense at infinity.

The graphs of the functions $\varphi, \tilde{\varphi}, \psi$, and $\tilde{\psi}$ in the case $N=2, L=2$ or 3 , are represented in Figure 3.

\section{REFERENCES}

[CD] A. COHEN AND I. DAUBECHIEs, Nonseparable bidimensional wavelet bases, preprint, Bell AT\&T Laboratories, Rev. Mat. Iberoamericana, to appear.

[CDF] A. Cohen, I. Daubechies, and J.C. Feauveau, Biorthogonal basis of compactly supported wavelets, Comm. Pure Appl. Math. 45 (1992), 485-560.

[CDM] A. Cavaretta, W. Dahmen, and C. Micchelli, Stationary Subdivision, Mem. Amer. Math. Soc. 93 (1991), \# 453.

[Co1] A. CoHEN, Ondelettes, analyses multirésolutions et filtres miroirs en quadrature, Ann. Inst. H. Poincaré Anal. Non Linéaire 7 (1990), 439-459.

[Co2] - "Biorthogonal wavelets" in Wavelets: A Tutorial in Theory and Applications, ed. by C. K. Chui, Academic Press, San Diego, 1992.

[CR] J.P. CONZE AND A. RAUGI, Fonctions harmoniques pour un operateur de transition et applications, preprint, Dept. de Math., Univ. de Rennes, 1990.

[Dau1] I. Daubechies, Orthonormal bases of compactly supported wavelets, Comm. Pure Appl. Math. 41 (1988), 909-996.

[Dau2] - Ten Lectures on Wavelets, CBMS-NSF Lecture Notes 61 SIAM, Philadelphia, 1992.

[E] T. EIROLA, Sobolev characterization of solutions of dilation equations, SIAM J. Math. Anal. 23 (1992), 1015-1030.

[La] W. LAWTON, Necessary and sufficient conditions for constructing orthonormal wavelets, J. Math. Phys. 32 (1991), 57-61.

[Me] Y. MEYER, Ondelettes et operateurs, Actualités mathématiques, Hermann, Paris, 1990.

[SB] M.J.T. SMITH AND T.P. BARNWELL, Exact reconstruction techniques for tree-structured subband coders, IEEE Trans. Acoust. Speech Signal Process. 34 (1986), 434-441.

[Ve] M. VetTERLI, Filter banks allowing perfect reconstruction, Signal Process. 10(1986), 219-244.

[Vi] L. VILLEMOES, Energy moments in time and frequency for two-scale difference equation solutions and wavelets, preprint, Math. Institute, Univ. of Denmark, 1991.

[VH] M. VeTterli AND C. HeRLeY, Wavelets and filter banks: theory and design, to appear in IEEE Trans. Acoust. Speech Signal Process.

Cohen: Ceremade, Université Paris-Dauphine, Paris, France

Daubechies: Rutgers University, NeW Brunswick, NeW JeRsey 08903; AT\&T Bell Laboratories, MurRay Hill, NeW JerSEy 07974 